\title{
Enhancement of Odor Avoidance Regulated by Dopamine Signaling in Caenorhabditis elegans
}

\author{
Koutarou D. Kimura, ${ }^{1,2,3}$ Kosuke Fujita, $^{2}$ and Isao Katsura ${ }^{1}$ \\ ${ }^{1}$ Structural Biology Center, National Institute of Genetics, Research Organization of Information and Systems, and Department of Genetics, Graduate \\ University for Advanced Studies, Shizuoka 411-8540, Japan, ${ }^{2}$ Department of Biological Sciences, Osaka University, Osaka 560-0043, and ${ }^{3}$ PRESTO \\ (Precursory Research for Embryonic Science and Technology), Japan Science and Technology Agency, Tokyo 102-0075, Japan
}

The enhancement of sensory responses after prior exposure to a stimulus is a fundamental mechanism of neural function in animals. Its molecular basis, however, has not been studied in as much depth as the reduction of sensory responses, such as adaptation or habituation. We report here that the avoidance behavior of the nematode Caenorhabditis elegans in response to repellent odors (2-nonanone or 1-octanol) is enhanced rather than reduced after preexposure to the odors. This enhancement effect of preexposure was maintained for at least $1 \mathrm{~h}$ after the conditioning. The enhancement of 2-nonanone avoidance was not dependent on the presence or absence of food during conditioning, which generally functions as a strong positive or negative unconditioned stimulus in the animals. These results suggest that the enhancement is acquired as a type of nonassociative learning. In addition, genetic and pharmacological analyses revealed that the enhancement of 2-nonanone avoidance requires dopamine signaling via $\mathrm{D}_{2}$-like dopamine receptor DOP-3, which functions in a pair of RIC interneurons to regulate the enhancement. Because dopamine signaling has been tightly linked with food-related information to modulate various behaviors of $C$. elegans, it may play different role in the regulation of the enhancement of 2-nonanone avoidance. Thus, our data suggest a new genetic and pharmacological paradigm for nonassociative enhancement of neural responses that is regulated by dopamine signaling.

\section{Introduction}

Plasticity in sensory responses is one of the fundamentals of neural function in animals. One simple form of such plasticity is nonassociative learning, an enhancement or reduction in sensory response after prior exposure to a stimulus in the absence of an unconditioned stimulus. In contrast to the intensive research on the reduction of sensory responses, as in adaptation or habituation (Thompson and Spencer, 1966), detailed studies of the enhancement of sensory responses have been mainly limited to sensitization in pain sensation (Millan, 1999) and defensive responses in the leech and Aplysia (Hawkins et al., 1993; Sahley, 1995). These sensitizations are induced by prior noxious stimuli, suggesting that they function to protect the animal from further disturbance. For innoxious chemical stimuli, enhanced odor re-

\footnotetext{
Received Dec. 4, 2009; revised Jan. 19, 2010; accepted 0ct. 5, 2010.

This work was supported by Grants-in-Aid for Scientific Research (A) (to I.K. and K.D.K) and (C) and Osaka University Life Science Young Independent Researcher Support Program through Special Coordination Funds for Promoting Science and Technology from the Ministry of Education, Culture, Sports, Science and Technology (MEXT), Precursory Research for Embryonic Science and Technology (PRESTO) from Japan Science and Technology Agency, and Transdisciplinary Incubation Fund from Research Organization of Information and Systems (to K.D.K). We thank H. Nakamura, A. Matsusue, H. Makino, A. Yamazoe, T. Takano-Shimizu, H. Kagoshima, R. Tsien, A. Fire, M. Koga, T. Ishihara, J.Umemori, P. Sengupta, A. Samuel, C. Rankin, A. Hart, S. Suo, and C. Bargmann for assistance, suggestions, and reagents, and 0 . Hobert for comments on the manuscript. We also thank M. Tomioka and Y. lino for kindly providing the C. elegans promoter library. Some nematode strains used in this work were provided by C. Bargmann, by the Caenorhabditis Genetics Center funded by the National Institutes of Health National Center for Research Resources, and by the National Bioresource Project funded by MEXT in Japan.

Correspondence should be addressed to Koutarou D. Kimura, Department of Biological Sciences, Osaka University, 1-1 Machikaneyama, Toyonaka, 0saka 560-0043, Japan. E-mail: kokimura-lab@umin.ac.jp.

D0I:10.1523/JNEUROSCI.6023-09.2010

Copyright $\odot 2010$ the authors $\quad 0270-6474 / 10 / 3016365-11 \$ 15.00 / 0$
}

sponses after prior experience in mice and in human have been reported (Wysocki et al., 1989; Wang et al., 1993; Yee and Wysocki, 2001; Dalton et al., 2002). Although such types of enhancement of sensory responses are thought to serve a critical role in reproduction and/or survival, the molecular basis has not been thoroughly investigated.

To identify a new molecular basis for the enhancement of sensory response, we used a genetically tractable model animal. The nematode Caenorhabditis elegans is ideal for analyzing mechanisms of neural plasticity, including nonassociative and associative forms of learning, because of its molecular, physiological and behavioral characteristics (for review, see Bargmann, 2006; Giles et al., 2006). C. elegans has been used as a model for detailed studies of the reduction in sensory responses: habituation to mechanical stimuli and adaptation to attractive odors or to watersoluble repulsive stimuli (Colbert and Bargmann, 1995; Rose and Rankin, 2001; Suzuki et al., 2003; Hilliard et al., 2005; Kindt et al., 2007). In addition to habituation and adaptation, which are caused by preexposure, avoidance responses to nose touch and to the repulsive odor 1-octanol are also suppressed by starvation (Chao et al., 2004). In contrast, only one form of enhancement of sensory response has been studied in C. elegans - the response to a mechanical stimulus to the body (Rankin et al., 1990)—and its molecular mechanism has not yet been reported.

We report here that avoidance behavior of $C$. elegans to a repulsive odor 2-nonanone (Bargmann et al., 1993; Troemel et al., 1997) is enhanced rather than reduced by preexposure. Unlike many other examples of behavioral plasticity in C. elegans, where sensory responses are strongly modulated by the presence 
or absence of bacterial food ("feeding status") during conditioning, the enhancement of 2-nonanone avoidance is independent of feeding status during the preexposure period, suggesting that it is acquired as nonassociative learning. Furthermore, from genetic and pharmacological analyses, we show that dopamine signaling regulates the enhancement of 2-nonanone avoidance. Our data suggest a new role of dopamine signaling in C. elegans to regulate behavioral plasticity.

\section{Materials and Methods}

Strains. The techniques used for culturing and handling C. elegans were essentially as described previously (Brenner, 1974). In a systematic analysis to identify genes involved in the enhancement of 2-nonanone avoidance, the following strains were used: acy-1(nu329), bas-1(ad446), cat-1(e1111), cat-2(e1112), cat-4(e1141), che-2(e1033), ckk-1 (ok1033), cmk-1(oy21), crh-1(tz2), dgk-1(nu62), dop-1(vs100), dop-2 (vs105), dop-2(vs105); dop-1(vs100) dop-3(vs106), dop-2(vs105); dop3(vs106), dop-3(vs106), dpy-19(n1347)glr-2(ak10), dyf-3(m185), eat4(ky5), eat-7(ad450), egl-2(n601), egl-19(m582), egl-30(n686), egl-4 (n478), egl-8(n488), glr-1(n2461), goa-1(n1134), gpa-2(pk16)gpa-3 (pk35), gpa-2(pk16)odr-3(n1605), gpa-11(pk349), gpc-1(pk298), grk1(ok1239), grk-2(rt97), hen-1(tm501), lim-4(ky403), mgl-2(tm355), mod-5(n3314), nmr-1(ak4), ocr-2(ak47), odr-1(n1936), odr-2(n2145), odr-3(n1605), odr-3(n2150), osm-3(p802), osm-9(ky10), sdf-13 (ut192), ser-2(pk1357), tax-4(p678), tax-6(p675), tbh-1(ok1196), tdc1(ok914), tph-1(mg280), ttx-3(ks5), ttx-3(mg158), unc-2(e55), unc-25 (e156), unc-43 (e408). General information of each mutant is available at WormBase (http://www.wormbase.org). gpa-2(pk16)odr3(n1605), odr-3(n1605) and str-1::mec-4(d) (CX3551 Is[str-1p::mec4(d) lin-15(+)]V; lin-15(n765ts)X) are kind gifts from C. Bargmann (The Rockefeller University, New York, NY); $t t x-3(k s 5)$ and $t t x-3(m g 158)$ are kind gifts from Ikue Mori (Nagoya University, Nagoya, Japan) and Oliver Hobert (Columbia University, New York, NY), respectively; tbh1 (ok1196) and $t d c-1$ (ok914) were obtained from the C. elegans gene knock-out consortium; cat-2(tm2261), dop-2(tm1062), dop-3(tm1356) and $m g l-2(t m 355)$ were obtained from National Bioresource Project (Japan). Wild-type C. elegans variety Bristol strain (N2) and all the other strains were obtained from the Caenorhabditis Genetics Center (University of Minnesota, Minneapolis, MN). The tm alleles of cat-2, dop-2 and dop-3 and tbh-1(ok1196) were backcrossed with N2 either 2 times (cat-2 and $t b h-1)$ or 5 times (dop-2 and $d o p-3)$ before use. The dop-2(tm1062); dop-3(tm1356), crh-1(tz2);dop-3(tm1356) and dop-3(tm1356)tbh1(ok1196) double mutants were constructed using standard genetic techniques.

Behavioral assay. For cultivation of animals for the behavioral analyses, we used standard $6 \mathrm{~cm}$ nematode growth medium (NGM) agar plates (Sulston and Hodgkin, 1988). Each of the plates contained a lawn of $E$. coli strain OP50, which had been spread on the plate and allowed to grow for 2-3 $\mathrm{d}$ at room temperature; the plates were kept on a bench during the period to dry properly. For egg-laying, four adult wild-type animals per plate were kept for $7.5 \mathrm{~h}$ at $20^{\circ} \mathrm{C}$ and then removed. The plates were incubated at $20^{\circ} \mathrm{C}$ for $65-70 \mathrm{~h}$ until the beginning of the assay. In these conditions, $\sim 100$ synchronized young adult animals were grown on a plate, and each had laid a few to several eggs at the beginning of the assay. Because some mutant animals laid fewer eggs or grew more slowly than the wild-type animals did, the number of such animals for egg-laying (up to seven animals per plate) or incubation temperature (up to $22^{\circ} \mathrm{C}$ ) was increased to obtain a similar developmental stage to the wild-type animals.

The odor avoidance assay was performed in a similar way to a previous study (Troemel et al., 1997) with the following modifications. (1) To avoid complexity in interpreting changes in buffers and media during cultivation, conditioning and the assay, we used NGM agar plates for the conditioning and assay as well as the cultivation, and NGM buffer (same composition as NGM agar plates except for agar) for washing. Higher concentrations of agar were used $(1.8 \%$ for conditioning and $2.5 \%$ for assay) to prevent the animals from digging into the agar, which was caused by repulsive odor stimulation. The agar was Bacterial Culture Medium grade (Wako Pure Chemical). (2) To minimize the time spent on the washing step of the animals, which usually takes $>10 \mathrm{~min}$ in the regular procedure, we used a "mesh column": the wider end of a P1000 tip was burned briefly and pushed onto a nylon mesh (ø $32 \mu \mathrm{m}$ or $\varnothing 20$ $\mu \mathrm{m}$; NBC Inc.) to make a seal. Then, the mesh was cut at just outside of the tip, and the tip was cut at $\sim 1 / 3$ from the wider end to open the other side. The adult animals were washed off with NGM buffer from the cultivation plates, and put into the mesh column. The bottom of the mesh column was pushed onto folded Kimwipes (Kimberly-Clark Inc.) to rapidly remove the buffer. The animals were washed once with NGM buffer in the column, suspended with $\sim 50 \mu \mathrm{l}$ of NGM buffer, and $3 \mu \mathrm{l}$ of the suspension including $\sim 20-40$ animals was put on the center of a 9 $\mathrm{cm}$ NGM plate without bacteria. The buffer dried within $1.5 \mathrm{~min}$, and the assay began with spotting the odor in two spots (see Fig. $1 A$ ). The whole preparation step, from the beginning of the washing to the beginning of the assay was completed within $5 \mathrm{~min}$. Twelve minutes after the beginning of the assay, the assay was stopped by the addition of $\mathrm{CHCl}_{3}$ on the lid of each plate. Assays for each condition and each strain on one $\mathrm{d}$ were performed in triplet, and the assays were repeated for 2-4 d. (3) To calculate the avoidance index, the $9 \mathrm{~cm}$ plates were divided into 6 sectors (\#1-6) with equal width (see Fig. 1A). The numbers of the animals in each fraction were scored and an avoidance index was calculated using the formula: avoidance index $=\sum W_{i} N_{i} / N$, where $W_{i}$ is the weight of each sector $(-2.5,-1.5,-0.5,+0.5,+1.5$, and +2.5 for sections $\# 1-6$, respectively), $N_{i}$ is the number of animals in each sector, and $N$ is the total number of animals in all six sectors of the plate. The animals that did not move from the center of the plate were not scored. As the weights are proportional to the distance from the center of the plate to the center of each sector, the avoidance index represents an average distance of the animals' positions from the center line of the plate (see Fig. $1 \mathrm{~A}$, the line between sector 3 and 4 ). For example, one unit of the avoidance index corresponds to $14 \mathrm{~mm}, 1 / 6$ of the plate's diameter $(84 \mathrm{~mm})$. Therefore, indices 1.65 and 2.15 (the ones of the naive and the preexposed animals, respectively, shown in Fig. $1 \mathrm{~A}$, below) correspond, respectively, to 23.1 and $30.1 \mathrm{~mm}$ of their average movement from the center line of the plate, which are close to the numbers found in the video-based real-time tracking (see Fig. $4 A ; 23.7 \pm 1.1$ and $30.7 \pm 1.4$, respectively).

Preexposure was performed in a similar way to the adaptation assay, as described previously (Colbert and Bargmann, 1995) with a few modifications. Animals grown on three to six NGM plates were washed with NGM buffer as described above and transferred to three fresh NGM plates $(6 \mathrm{~cm})$ with or without bacteria. After drying the buffer, odorant was distributed on the surface of six NGM agar plugs (at the points of a pentagon plus the center) on the lid of a plate, and the plate was sealed with Parafilm. After 60-70 min (otherwise indicated) of incubation at room temperature, the animals were washed with NGM buffer and subjected to the avoidance assay. The amounts of odorants used for preexposure were 30 or $90 \mathrm{nl}$ ( $3 \mu \mathrm{l}$ of $1 \%$ or $3 \%$ ) of 1 -octanol, or 30 or $90 \mathrm{nl}(0.6$ $\mu \mathrm{l}$ of $5 \%$ or $15 \%)$ of 2 -nonanone per plate. Generally, the magnitudes of enhancement were not different when either amount of odor was used for preexposure.

The glycerol avoidance assay was performed using four-quadrant plates (Wicks et al., 2000) with $2.5 \%$ agar NGM $\pm 100 \mathrm{~mm}$ glycerol. Avoidance index was calculated as $(C-A) /(C+A)$, where $A$ and $C$ are the numbers of animals at the quadrants with or without glycerol, respectively. For preexposure to higher osmolarity, an NGM plate with $250 \mathrm{~mm}$ sucrose was used.

Automated tracking and analysis of run/pirouette. In the standard worm-tracking system (Feng et al., 2004), the position of the assay plate is motor-controlled to maintain the animal in a visual field of a microscope. Since this could disturb the odor gradient in our avoidance assay, we used a high-resolution video camera in a fixed position to capture the whole area of an assay plate without movement while tracking individual animals on the plate. This method was originally reported by Colosimo et al. (2004) and modified in our study as follows. The plate was illuminated by a high-density red LED ring array LDR2-170RD-LA (CCS Inc.), and video images of surface of the entire plate were captured at the rate of 1 frame per $2 \mathrm{~s}(0.5 \mathrm{~Hz})$ by a color digital camera DFW-X710 (Sony) with a resolution of $1024 \times 768$. With this system, individual animals can be identified as $5 \sim 10$ pixels with higher intensity than the background im- 
age. Several animals were put on a $9 \mathrm{~cm}$ NGM assay plate and tracked simultaneously for the analysis. The camera images were transferred to a Windows PC with a video acquisition card CFW-46 (Technoscope Co. Ltd.) and acquisition software VFS-42 (Chori Imaging Corp.) via IEEE1394 cable and stored as BMP files.

Definition of runs and pirouettes. If we used the original definition of run as locomotion of $|d \theta / d t| \leq 50^{\circ} \mathrm{s}^{-1}$ persisting $>6.05 \mathrm{~s}$ (PierceShimomura et al., 1999), each of the periods of frequent turning during the 2-nonanone avoidance (see Fig. $4 C$, indicated by black) would be divided into multiple short "runs" and "pirouettes" in most of the cases. This discrepancy may be due to differences between chemotaxis toward an attractive soluble compound, such as $\mathrm{NH}_{4} \mathrm{Cl}$, and avoidance responses to a repulsive odor. Therefore we redefined runs and pirouettes in 2-nonanone avoidance. We first discriminated the two patterns manually based on the shape of the tracks, and subsequently we searched for parameters that mathematically separate them most appropriately. As a result, we defined "run" as the following situation if it continued for $14 \mathrm{~s}$ or longer: (1) $|d \theta|$ during 2 frames $<100^{\circ}$ and (2) velocity during 2 frames $>0.05 \mathrm{~mm} / \mathrm{s}$; all other situations were classified as "pirouette." Durations and numbers of runs and pirouettes were scored after 2 min of the assay because the animals did not appear to initiate avoidance behavior during the first $2 \mathrm{~min}$ as shown in Figure $4 \mathrm{~A}$. This delay may reflect the time for diffusion of 2-nonanone from the source.

Transgenic rescue of mutant phenotype. Germline transformation was performed by microinjection (Mello et al., 1991). The injection solution contained the DNA to be tested ( 5 or $50 \mathrm{ng} / \mu \mathrm{l}), 1 \mathrm{ng} / \mu \mathrm{l}$ myo-3p::mRFP as a marker, and PvuII-cut N2 genomic DNA or pBR322 plasmid DNA as a carrier, for a total DNA concentration of $100 \mathrm{ng} / \mu \mathrm{l}$. myo-3 promoter was from pPD115.44 (a gift from A. Fire, Stanford University, Palo Alto, CA), and mRFP was a gift from R. Tsien (University of California, San Diego, CA). Because the transgenes were maintained as extrachromosomal arrays and not all progenies contained the transgene, the animals were assayed as a mixed population of transgenic and nontransgenic animals, and only the $\mathrm{RFP}(+)$ animals were scored. Although the expression of myo-3p::mRFP somewhat affects basal locomotion (for example, compare the naive and the mock-treated wild-type animals in Fig. $7 \mathrm{~A}$ with those of other figures), it does not affect the enhancement of 2-nonanone avoidance as well as the animal's other behavioral plasticity (data not shown).

Molecular biology. For the cat-2 rescue experiments, the wild-type cat-2 genomic fragment, including $2641 \mathrm{bp}$ upstream and $1783 \mathrm{bp}$ downstream sequences, was amplified by PCR and used for injection at 5 $\mathrm{ng} / \mu \mathrm{l}$. For the $d o p-3$ rescue experiments, dop-3 cDNA (a gift from S. Suo, Mt. Sinai Hospital, Toronto, ON, Canada) was inserted into pPD-DEST2 vector (Tomioka et al., 2006) to generate a destination vector pDESTdop-3 for the GATEWAY system (Invitrogen). pENTR plasmids containing the promoter sequences for H20 (a gift from T. Ishihara, Kyushu University, Fukuoka, Japan), acr-2 (Hallam et al., 2000), glr-2 (Brockie et al., 2001), lin-11 (Hobert et al., 1998), odr-2 (Chou et al., 2001), tax-2 (Coburn and Bargmann, 1996), unc-47 (Eastman et al., 1999), unc-86 (Finney and Ruvkun, 1990) were kindly provided by M. Tomioka and Y. Iino (University of Tokyo, Tokyo, Japan) and subjected to site-specific recombination with pDEST-dop-3. ceh-17p::dop-3 cDNA and tbh-1p::dop-3 cDNA were gifts from S. Suo (Suo et al., 2009).

Drug treatment. Haloperidol (10 mм; Sigma), loxapine (5 mm; Sigma), or SCH23390 ( $R$-(+)-7-chloro-8-hydroxy-3-methyl-1-phenyl-2,3,4,5tetrahydro- $1 \mathrm{H}$-3-benzazepine) (10 mm; Sigma) was dissolved in $1 \%$ ascorbic acid (haloperidol) or in $\mathrm{dH}_{2} \mathrm{O}$ (other drugs). The $1 \%$ ascorbic acid or $\mathrm{dH}_{2} \mathrm{O}$ was used as a non-drug control. All the solutions contained $250 \mu \mathrm{g} / \mathrm{ml}$ streptomycin to suppress contamination of other bacteria during the preparation. The drug solution $(0.1 \mathrm{ml})$ was spread onto NGM agar plates and left for 10-20 min to dry. For plates that were used for incubation before the preexposure to 2-nonanone, $50 \mu \mathrm{l}$ of concentrated bacteria OP50 [grown in $100 \mathrm{ml}$ of LB medium for overnight at $37^{\circ} \mathrm{C}$ and then spun down and suspended in $2 \mathrm{ml}$ of $\mathrm{dH}_{2} \mathrm{O}$ (Colbert and Bargmann, 1995)] with $250 \mu \mathrm{g} / \mathrm{ml}$ streptomycin was mixed with an equal amount of the drug solution, and poured onto the drug plate. The next day, the animals that were cultivated on regular NGM plates were transferred to the drug plates with OP50 and kept at $20^{\circ} \mathrm{C}$ for $3-5 \mathrm{~h}$. Then the animals were directly used for the avoidance assay (the naive animals), or transferred to the drug plates without OP50 for the preexposure.

High concentrations of drugs were used because of the impermeability of the C. elegans cuticle, and the concentrations used in this study were not different from previous studies. For example, $20 \mathrm{~mm}$ dopamine (3 $\mathrm{mg} / \mathrm{ml}$ ) was used to observe its effects on wild-type C. elegans (Schafer and Kenyon, 1995). In addition, in a previous study (Weinshenker et al., 1995), dopamine antagonists were spread on the surface of an NGM agar plate in the amount of $0.7 \mathrm{ml}$ at $0.75 \mathrm{mg} / \mathrm{ml}(=0.53 \mathrm{mg} /$ plate $)$, which is similar in value to the ones in our study. For example, the amount of 10 $\mathrm{mM}(=3.8 \mathrm{mg} / \mathrm{ml})$ haloperidol used per plate was $0.1 \mathrm{ml}(0.38 \mathrm{mg}$ for preexposure) or $0.125 \mathrm{ml}$ ( $0.47 \mathrm{mg}$ for prior cultivation with food).

Statistical analysis. Each avoidance index was calculated from nine independent avoidance assays unless otherwise indicated; in general, $20-40$ animals were used in one plate assay, and therefore $\sim 200-300$ animals in the nine assays were used to calculate the mean avoidance index $( \pm$ SEM $)$ in the figure. Multiple comparisons of avoidance indices were performed using Prism version 5.0 for Mac OSX (GraphPad Software). For comparisons of avoidance indices among naive, mocktreated, and preexposed animals of one strain or one condition (i.e., one set of three adjacent columns), one-way ANOVA with post hoc Tukey's test (all except for Fig. 5D; see below) or Newman-Keuls' test (used in Fig. $5 D$ ) was used. For comparisons of the indices of each condition between the wild-type and the dop mutants (see Fig. $6 \mathrm{~B}$ ), ANOVA with post hoc Dunnett's test was used. For single comparisons, statistical significance was determined by a two-tailed $t$ test.

\section{Results \\ Odor avoidance is enhanced by preexposure to repulsive odors}

We investigated, for the first time to our knowledge, the plasticity of avoidance behavior to odors after prior exposure in C. elegans. Because repulsive signals are signs for danger or unsuitable conditions to survive or to reproduce, we speculated that behavioral plasticity other than reduction of sensory response may exist. We used 2-nonanone and 1-octanol as representative repulsive odors for two reasons. First, these two odors are among 10 repulsive odors identified from 121 volatile compounds tested for chemotaxis on C. elegans (Bargmann et al., 1993). Second, further investigation showed that, under similar experimental conditions to ours, 2-nonanone and 1-octanol are mainly sensed by two pairs of sensory neurons: AWB and ADL, respectively (Troemel et al., 1997).

We first studied avoidance of the repulsive odor 2-nonanone. Unlike 1-octanol avoidance (Chao et al., 2004), we found that starvation for $1 \mathrm{~h}$ did not significantly affect 2-nonanone avoidance (Fig. $1 B$, lanes 1 vs 2 , and 4 vs 5 ). In contrast, after the animals were preexposed to 2-nonanone for $1 \mathrm{~h}$, the preexposed animals unexpectedly migrated farther away from the odor source than the naive or the mock-treated animals did in the assay (Fig. 1 A, B, lanes 1-3; a detailed time course analysis of this behavioral change is described in Fig. 4). The enhanced response in 2-nonanone avoidance was observed when $600 \mathrm{nl}$ or $200 \mathrm{nl}$, but not $60 \mathrm{nl}$, of 2-nonanone was used as an odor source in the assay (Fig. $1 B$ ). These results indicate that, unlike other stimuli that cause adaptation or habituation after preexposure (Colbert and Bargmann, 1995; Rose and Rankin, 2001; Suzuki et al., 2003; Hilliard et al., 2005; Kindt et al., 2007), the repulsive odor 2-nonanone causes enhancement of avoidance behavior after preexposure.

We next studied the effect of preexposure to another repulsive odor, 1-octanol, to test whether 1-octanol and 2-nonanone have a similar effect on avoidance behavior. We found that $1 \mathrm{~h}$ of starvation suppressed the 1-octanol avoidance response (Fig. 1C, lanes 1 vs 2) as in the case of immediate avoidance response to a 

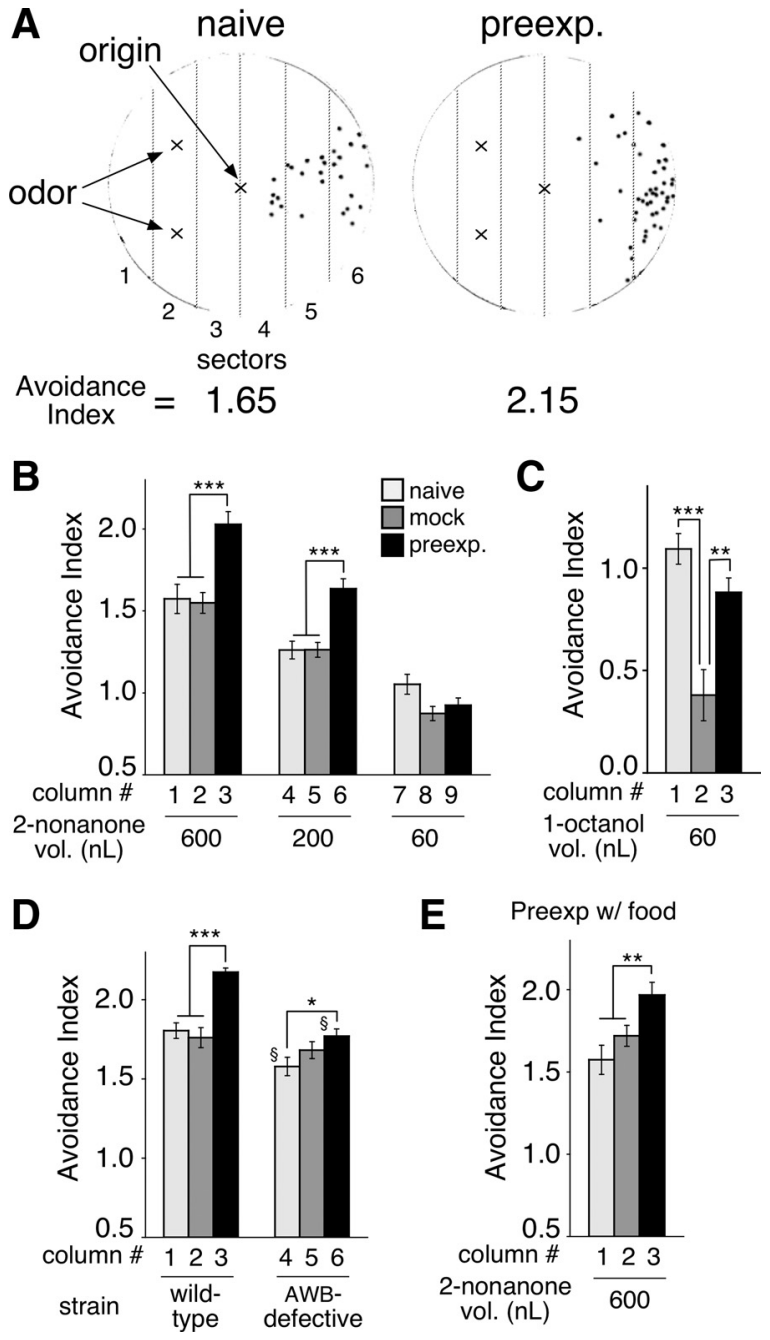

Figure 1. Preexposure causes enhancement of odor avoidance. A, Typical examples of avoidance assay of the naive and the preexposed animals to $600 \mathrm{nl}$ of 2-nonanone. Black dots indicate positions of the end points of animals' avoidance behavior. Positions of the odor source, the original position of animals and lines between the sectors were overlaid. $\boldsymbol{B}$, Avoidance index of 2-nonanone avoidance after preexposure. The naive animals ("naive") or animals kept in the absence ("mock") or presence ("preexp.") of $30 \mathrm{nl}$ of 2-nonanone in NGM plates without food for $1 \mathrm{~h}$ were subjected to the avoidance assay with 600,200 or $60 \mathrm{nl}$ of 2-nonanone. The avoidance index reflects an average of avoidance distances of the animals from the center line of the plate. Open, gray, and solid bars indicate results of naive, mock-treated and preexposed animals, respectively, and each bar represents the mean \pm SEM of 9 independent experiments throughout the figures unless otherwise indicated. C, 1-0ctanol avoidance after preexposure. The animals were preexposed to $90 \mathrm{nl}$ of 1-octanol without food for $1 \mathrm{~h}$, and subjected to an avoidance assay with $60 \mathrm{nl}$ of 1-octanol. D, The enhancement of 2-nonanone avoidance is AWBdependent. Animals were preexposed to $90 \mathrm{nl}$ of 2-nonanone in the absence of food for $1 \mathrm{~h}$ and then assayed with $600 \mathrm{nl}$ of 2-nonanone. § indicates statistical difference from the corresponding wild-type index (see Results). $E$, Preexposure in the presence of food also caused the enhancement of 2-nonanone avoidance. The animals were preexposed to $30 \mathrm{nl}$ of 2-nonanone in the presence of food and assayed with $600 \mathrm{nl}$ of the odor. ${ }^{*} p<0.05,{ }^{* *} p<0.01$ and ${ }^{* * *} p<$ 0.001 throughout figures.

close presentation of the odor (Chao et al., 2004). When the animals were preexposed to 1-octanol during the $1 \mathrm{~h}$ starvation period, however, the avoidance behavior was not suppressed, and the animals exhibited 1-octanol avoidance at a similar magnitude to the naive animals (Fig. 1C). This is a good contrast to the result of ASH-mediated higher osmolarity avoidance, where preexposure to higher osmolarity caused suppression of subsequent osmolarity avoidance (Fig. $2 \mathrm{~A}$, left). These results suggest that the
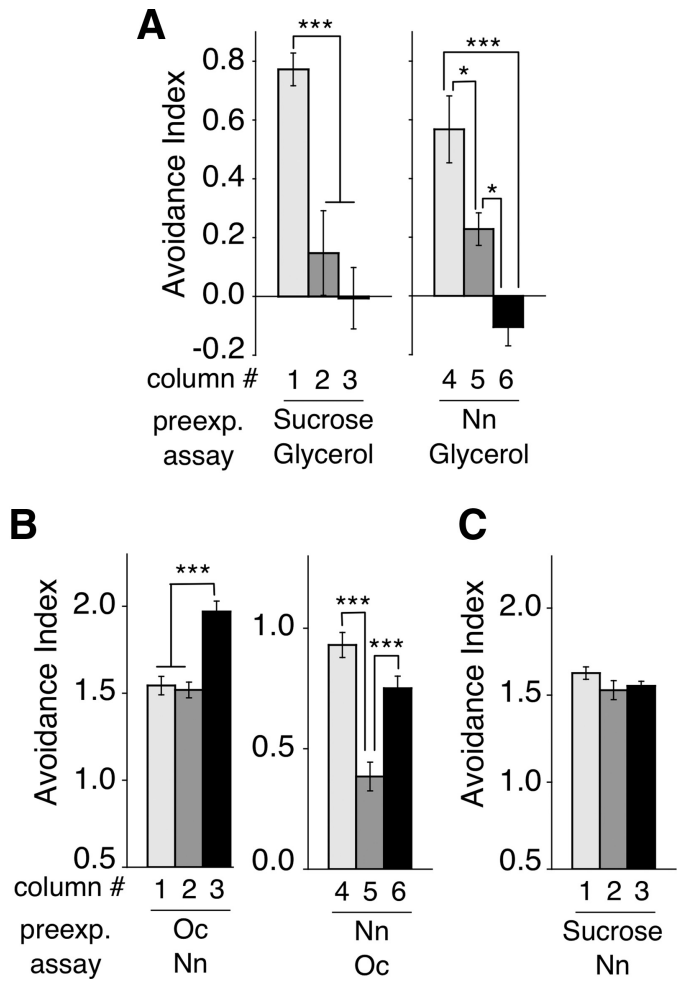

Figure 2. Preexposure is effective across different repulsive odors. $\boldsymbol{A}$, The animals were preexposed to $250 \mathrm{~mm}$ sucrose (left) or to $90 \mathrm{nl}$ of 2-nonanone (right) in the absence of food for $1 \mathrm{~h}$ and assayed with $100 \mathrm{~mm}$ glycerol in quadrant plates for $15 \mathrm{~min}$. Glycerol avoidance was partially suppressed by starvation (lane 2 and 5 ) and further suppressed by the preexposure to 250 mm sucrose (lane 3) or to 2-nonanone (lane 6). We used sucrose for preexposure (left panel) to confirm that higher osmolarity itself affected the subsequent osmolarity avoidance. Each bar represents the mean \pm SEM of 6 independent experiments. $\boldsymbol{B}$, The animals were preexposed to $90 \mathrm{nl}$ of 1-octanol ( $0 \mathrm{c}$ ) and assayed with $600 \mathrm{nl}$ of 2-nonanone ( $\mathrm{Nn}$ ) (left panel) or preexposed to $30 \mathrm{nl}$ of 2-nonanone and assayed with $60 \mathrm{nl}$ of 1-octanol (right panel). Each bar represents the mean \pm SEM of 6 independent experiments. $\boldsymbol{C}$, The animals were preexposed to $250 \mathrm{~mm}$ sucrose and assayed with $600 \mathrm{nl}$ of 2-nonanone. Each bar represents the mean \pm SEM of 9 independent experiments.

effect of preexposure to 1-octanol either shares the same mechanism with the enhancement of 2-nonanone avoidance, or it is caused by reversal or suppression of the starvation-induced suppression of 1-octanol avoidance, which is not correlated with the enhancement of 2-nonanone avoidance.

To investigate whether the preexposure-induced changes in 2-nonanone and 1-octanol avoidance are stimulus-specific, we preexposed the animals to 2-nonanone, 1-octanol or higher osmolarity for $1 \mathrm{~h}$ in the absence of food, and then subjected the animals to an avoidance assay to each stimulus. Preexposure to 2-nonanone caused enhancement of 1-octanol avoidance, and vice versa (Fig. $2 B$ ). However, reduction was caused by the 2-nonanone preexposure in higher osmolarity avoidance (Fig. $2 A$, right). In addition, preexposure to higher osmolarity did not enhance 2-nonanone avoidance (Fig. 2C). These results suggest that the effect of preexposure on odor avoidance is not odorspecific; that is, the information from the preexposure to repulsive odors is shared between either of the odor responses, but not with other sensory modalities.

Although 2-nonanone avoidance is reportedly mediated by AWB sensory neurons (Troemel et al., 1997), other sensory neuron(s) or non-neuronal tissue could contribute to the odor sensation at least in certain conditions, as in the case of immediate 1-octanol avoidance (Chao et al., 2004). To investigate whether 
AWB sensory neurons are in fact responsible for the enhancement of 2-nonanone avoidance, we used transgenic animals expressing the toxic gain-of-function degenerin gene $m e c-4(d)$ to cause a specific defect in AWB neurons (Driscoll and Chalfie, 1991; Troemel et al., 1997). AWB-mediated 2-nonanone avoidance, as well as dye-uptake, are significantly, but not completely, affected in these animals. The naive avoidance response to 2-nonanone was partially affected by the AWB-defect as reported previously (Troemel et al., 1997) (Fig. $1 D$, lanes 1 vs $4 ; p<0.05$ ); the partial defect could be explained either by the residual AWB function or by the contribution of other sensory neurons. More severely affected was the enhancement of 2-nonanone avoidance: the avoidance response after 2-nonanone preexposure was significantly smaller in the AWB-defective animals compared with the wild-type animals (lanes 3 vs $6 ; p<0.01$ ) and was comparable to the response after the mock-treatment of the AWB-defective animals (lanes 5 vs $6 ; p>0.1$ ). Although there was a statistical difference between the naive and the preexposed AWB-defective animals (lanes 4 vs $6, p<0.05$ ), no statistical difference was found between the mock-treated and the preexposed animals, suggesting that the difference was not caused by the 2-nonanone preexposure, but by starvation or other factors during the conditioning. Thus, our results suggest that the AWB neurons are the major sensory neurons responsible for the enhancement of 2-nonanone avoidance. Our results may also suggest that the naive 2-nonanone avoidance itself is likely mediated by AWB neurons as well as other sensory neurons in our experimental conditions.

The enhancement of 2-nonanone avoidance is independent of feeding status during conditioning

Most types of behavioral plasticity in C. elegans are affected by feeding status. Within minutes of entering or being removed from a bacterial lawn, changes are seen in C. elegans behaviors such as defecation, egg-laying, pharyngeal pumping and speed and direction of locomotion (Horvitz et al., 1982; Avery and Horvitz, 1990; Liu and Thomas, 1994; Sawin et al., 2000; Hills et al., 2004; Gray et al., 2005). Moreover, after the animals have been preexposed to a particular stimulus in the presence or absence of food, their behavior to the stimulus is modulated more positively or negatively, respectively, in the subsequent assay (Bargmann, 2006; Giles et al., 2006). For example, preexposure to $\mathrm{NaCl}$ in the absence of food for $1 \mathrm{~h}$ leads to salt avoidance learning (Tomioka et al., 2006). Therefore, it was possible that the enhancement of 2-nonanone avoidance could be caused by association between 2-nonanone and starvation, which functioned as a negative unconditioned stimulus. However, preexposure to the odor in the presence of food also caused significant enhancement (Fig. 1E). Although the magnitude of the enhancement appears larger after the animals were preexposed in the absence of food (Fig. $1 B$, lanes 1-3), the differences in magnitude between the presence and absence of food during conditioning were not consistent (see also lanes $1-3$ in Fig. $5 B, C$ ). Together, these results suggest that this behavioral plasticity does not require either the presence or absence of food as an unconditioned stimulus.

\section{The effect of preexposure is memorized for at least $\mathbf{1 ~ h}$}

We next investigated whether the enhancement of 2-nonanone avoidance is stored as a "memory." Even after the animals had been preexposed to 2-nonanone in the absence of food and subsequently kept in the absence of food and odor for an additional $1 \mathrm{~h}$, they also exhibited the enhancement of 2-nonanone avoidance (Fig. 3B). This result suggests that the "memory" of the
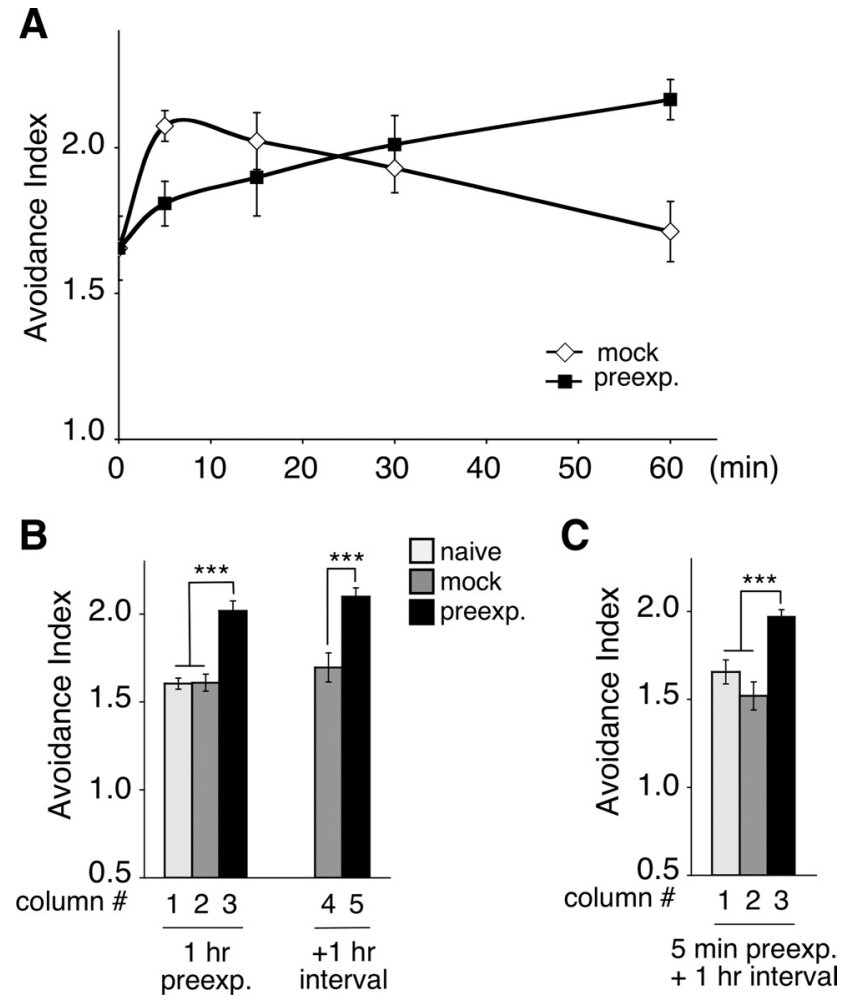

Figure 3. Memory of 2-nonanone preexposure. $\boldsymbol{A}$, The animals were mock-treated (open circle) or preexposed to $30 \mathrm{nl}$ of 2-nonanone (solid circle) for $0,5,15,30$ or $60 \mathrm{~min}$ and assayed with $600 \mathrm{nl}$ of 2-nonanone. Each circle represents the mean \pm SEM of 6 independent experiments. $\boldsymbol{B}$, The animals were mock-treated or preexposed to $30 \mathrm{nl}$ of 2-nonanone, and assayed with $600 \mathrm{nl}$ of 2-nonanone immediately after (lanes 1-3) or after a $1 \mathrm{~h}$ interval in the absence of food and the odor (lanes 4, 5). Each bar represents the mean \pm SEM of 6 independent experiments. $\boldsymbol{C}$, The animals were preexposed to $90 \mathrm{nl}$ of 2 -nonanone for 5 min and then kept in the absence of food and the odor before the assay with $600 \mathrm{nl}$ of 2-nonanone. Each bar represents the mean \pm SEM of 6 independent experiments.

preexposure may be stored for at least $1 \mathrm{~h}$. In addition, the formation of "memory" required 30-60 min: when the animals were preexposed to 2 -nonanone for $5,15,30$, or $60 \mathrm{~min}$, the magnitude of the enhancement gradually increased (Fig. $3 A$ ). In the mock-treated animals, the avoidance index transiently increased shortly after the beginning of the conditioning and decreased to the basal level by $60 \mathrm{~min}$. This transient increase could be caused by starvation or by exposure to a small amount of the solvent ethanol ( $3 \mu$ l per plate). Interestingly, when the animals were preexposed to 2-nonanone for $5 \mathrm{~min}$ and then kept in the absence of food and the odor for $1 \mathrm{~h}$, they exhibited the enhancement, although 5 min of preexposure itself did not (Fig. $3 C$ vs 5 min in Fig. $3 A$ ). The fact that the "memory" required $\sim 30$ min or more to be formed and was maintained at least for $1 \mathrm{~h}$ together raises the possibility that the effect of preexposure may be maintained as a middle-term memory (DeZazzo and Tully, 1995).

\section{Run duration, but not migration velocity, is enhanced by the preexposure}

How does the "enhancement" of 2-nonanone avoidance occur? Is it a simple increase in migration velocity? To obtain a strategic insight for regulation of the enhancement of 2-nonanone avoidance, we used a video-based tracking system. We measured the positions of the animals and found that for naive and mocktreated animals, the average distance from the center line increased at similar rates after $2 \mathrm{~min}$ of the assay, whereas that for 2-nonanone- 
preexposed animals increased $\sim 30 \%$ faster (0.037, 0.041 and $0.051 \mathrm{~mm} / \mathrm{s}$, respectively; Fig. 4A). These results could support the idea that the preexposed animals move faster than the control animals, which leads to a longer migration distance in the assay. However, the average velocities, regardless of locomotion direction of the animals, were not significantly different among the naive, mock-treated and preexposed animals (Fig. 4B). How, then, does the "enhancement" occur?

We considered the possibility that some change might occur in the mode of the animals' movement, such as pirouettes and runs (Pierce-Shimomura et al., 1999); pirouettes are periods with frequent changes in direction, and runs are periods of relatively long, straight movement. The tracks of the animals showed that the 2-nonanone avoidance pattern is also composed of runs and pirouettes (Fig. 4C). We then found that the preexposure caused significant increases in the average duration of runs, but not in pirouettes (Fig. 4D). Further, in histograms of run duration, we found interesting differences between the control (i.e., naive and mock) and the preexposed animals (Fig. 4E): in the preexposed animals, the number of longer runs ( $\geq 92 \mathrm{~s}$ duration) was larger and that of shorter runs (18-24 s duration) was smaller, whereas the number of 14-16 s runs remained unchanged. These results suggest that, at the behavioral level, the enhancement of 2-nonanone avoidance is caused primarily by increases in run duration.

\section{Dopamine signaling is required for the} enhancement of 2-nonanone avoidance To identify genes involved in the enhancement of 2-nonanone avoidance, we investigated the phenotype of various mutant lines of genes involved in neural functions (see Materials and Methods). From the analysis, we found that the mutants for biosynthesis and transmission of dopamine (Loer and Kenyon, 1993; Duerr et al., 1999; Lints and Emmons, 1999; Sze et al., 2000; Ranganathan et al., 2001) were defective in the enhancement of 2-nonanone avoidance (Fig. 5A). The enhancement of 2-nonanone avoidance was significantly affected by mutations in cat4(e1141), cat-2(e1112), bas-1(ad446) and cat-1(e1111), the genes required for dopamine specifically or for dopamine and serotonin, but not by tph-1(mg280), which is specifically required for serotonin. In addition, cat-2(tm2261), another allele of the gene specifically required for dopamine synthesis, also exhibited a significant decrease in the enhancement of 2-nonanone avoidance after conditioning in the absence as well as in the presence of food (Fig. $5 B, C$ ). Moreover, the phenotype was rescued by the introduction of a wild-type genomic fragment into the cat-2 mutants (Fig. 5D). Together, all of these results suggest that dopamine signaling is required for the enhancement of 2-nonanone avoidance.
E
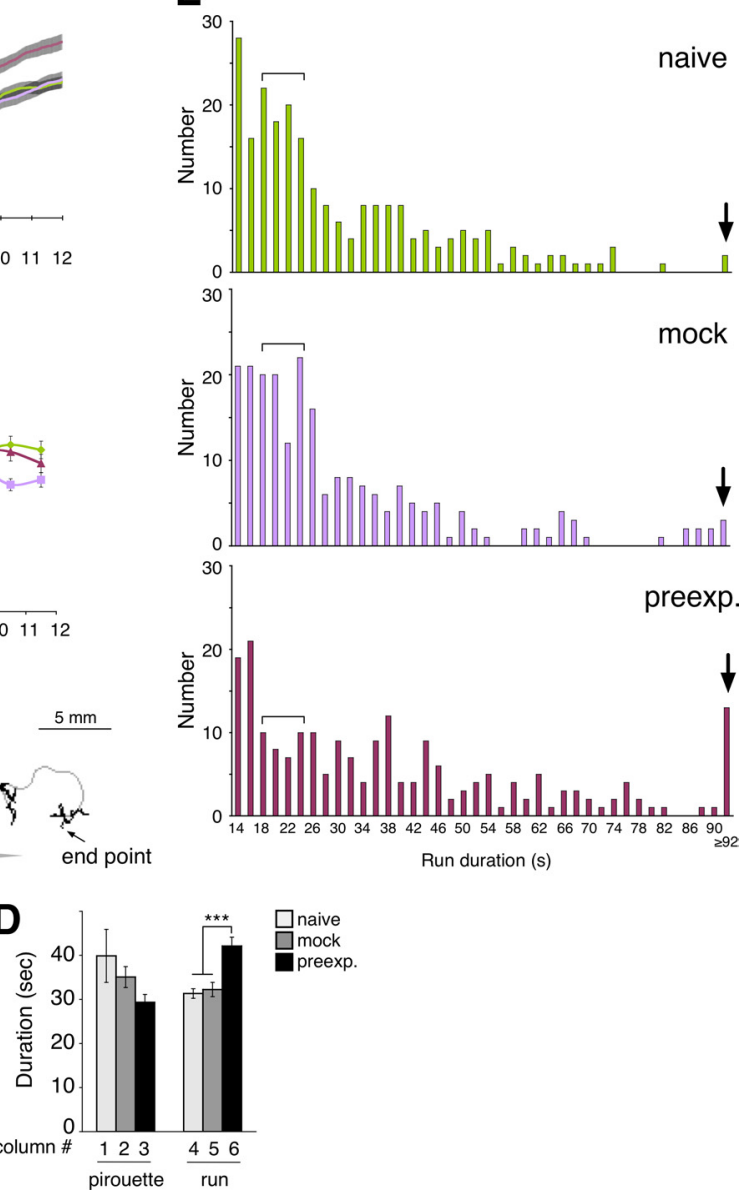

Figure 4. Run duration is increased by preexposure. $\boldsymbol{A}$, Time course of changes in animals' average position during 2-nonanone avoidance. Distance of each animal's centroid from the center line of the assay plate (the line between sectors 3 and 4 in Fig. $1 \mathrm{~A}$ ) was averaged for each frame and plotted as a function of time. Twenty-five animals were used for each condition to calculate the . Gray area indicates the SEM. B, Time course changes of animals' velocity. Velocity regardless of locomotion direction animals' run duration ( 25 animals each). The long run ( $\geq 92 \mathrm{~s}$ duration) and the shorter run (18 $-24 \mathrm{~s}$ duration) are indicated by a black arrow and a bracket, respectively.

To further confirm the involvement of dopamine signaling, we conducted pharmacological analysis with dopamine receptor antagonists (Waddington and O'Boyle, 1989). Dopamine receptors are classified into $\mathrm{D}_{1}$ - and $\mathrm{D}_{2}$-subtypes, and they function oppositely to regulate intracellular signaling, such as up- and downregulation of cAMP signaling, respectively (Missale et al., 1998). We found that haloperidol, a $\mathrm{D}_{2}$-specific antagonist also used as antipsychotic drug, specifically suppressed the enhancement of preexposed animals (Fig. 6A, lanes 13-15). Structurally unrelated $\mathrm{D}_{1} / \mathrm{D}_{2}$ antagonist and an antipsychotic loxapine also completely suppressed the enhancement compared with the mock-treated control animals (lanes 8 vs 9 ). Suppression of the naive response to 2-nonanone by the drug (lane 7) may be caused by its broader spectrum. The $\mathrm{D}_{1}$ antagonist $\mathrm{SCH} 23390 \mathrm{did}$ not affect the enhancement of 2-nonanone avoidance (lanes 4-6). These results suggest that a subclass of dopamine receptor(s) is required for the enhancement of 2-nonanone avoidance. Because the drugs were only applied to adult animals, the results also suggest that dopamine signaling is required in the developed 
A
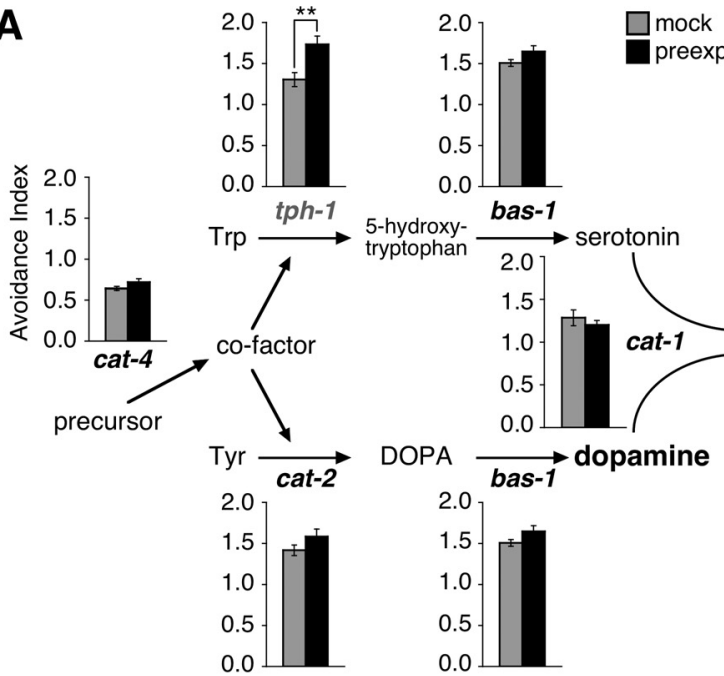

B
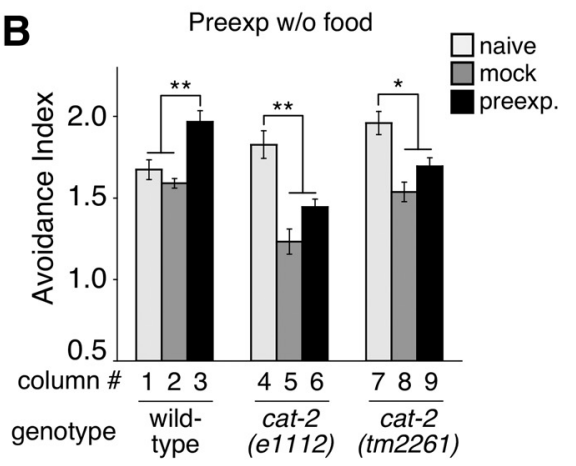

C

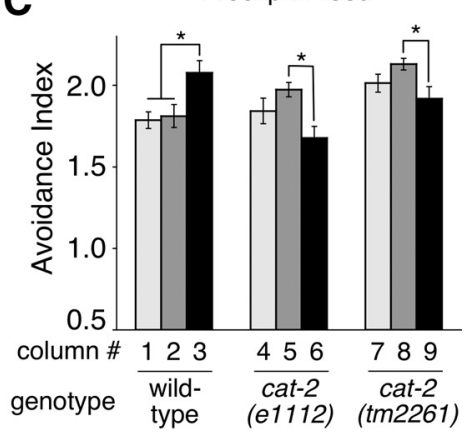

D

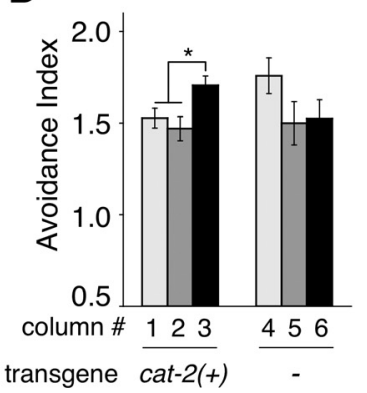

Figure 5. Enhancement of 2-nonanone avoidance is regulated by dopamine signaling. $\boldsymbol{A}$, Monoamine biosynthetic pathways and the phenotype of mutants in the pathways. The avoidance of naive animals was not analyzed to save time during the series of analyses. Names of the mutants defective in the enhancement of 2-nonanone avoidance are shown in bold black letters, and the one without the defect is shown in gray. $\boldsymbol{B}, \boldsymbol{C}$, The animals were preexposed to $90 \mathrm{nl}$ of 2 -nonanone in the absence $(\boldsymbol{B})$ or presence $(C)$ of food and then assayed with $600 \mathrm{nl}$ of 2 -nonanone. $D$, Wild-type genomic fragment of a cat-2 gene at $5 \mathrm{ng} / \mu$ l was introduced into cat-2(tm2261) mutants, and transgenic animals were preexposed to $90 \mathrm{nl}$ of 2-nonanone in the absence of food and assayed with $600 \mathrm{nl}$ of 2-nonanone. Two independent transgenic lines were used for each of cat-2(+) and control genotypes. Each bar represents the mean \pm SEM of 12 independent experiments.

nervous system for the enhancement of odor avoidance, but not for the development of it.

We then identified one dopamine receptor homolog that is required for the enhancement of 2-nonanone avoidance. In $C$. elegans, one $\mathrm{D}_{1}$-like (dop-1) and two $\mathrm{D}_{2}$-like (dop-2 and dop-3) receptors have been identified (for review, see Chase and Koelle, 2007). Two alleles of dop-2 mutants as well as dop-1 mutants exhibited significant enhancement of 2-nonanone avoidance, just as wild-type animals did (Fig. $6 B$ ). In contrast, all of the dop-3 mutants and dop-2;dop-3 double mutants showed the following abnormal phenotypes. (1) The avoidance indices of the preexposed animals were not significantly different from those of the naive animals in each genotype. (2) The avoidance index of the preexposed dop-3 or dop-2;dop-3 animals was statistically smaller than that of the preexposed wild-type animals (ANOVA with Dunnett's post test; $p>0.05$ for dop-1 (vs101), dop-2(tm1062) and dop-2(vs105); $p<0.01$ for dop-3(tm1356), dop-3 (vs106), dop-2(tm1062);dop-3(tm1356) and dop-2(vs105);dop-3(vs106)), whereas no differences were found between wildtype and mutant animals in the naive condition (ANOVA with Dunnett's post test; $p>0.05$ for any mutants). The differences in indices between the alleles of the same gene may be caused by side mutation(s) in each of the mutant strains. Although the incomplete suppression of enhancement by dop-3 mutation may suggest the involvement of another dopamine receptor, invertebrate-specific dopamine receptor homolog dop-4(tm1392) (Sugiura et al., 2005) did not appear to be involved as a single mutation or double mutation with dop-3 (K. F. and K. D. K., unpublished observation). Together, these results suggest that $\mathrm{D}_{2}$-like dop-3 plays a significant role in the enhancement of 2-nonanone avoidance.

\section{dop-3 functions in RIC interneurons to regulate the enhancement}

Where and how does the dopamine signaling function to regulate the enhancement of 2-noanone avoidance? To identify the site-of-action of the dop-3 receptor, we conducted cell-specific rescue experiments of the enhancement of the 2-nonanone avoidance phenotype by expressing the $d o p-3$ cDNA with various neuronal promoters in the dop-3 mutants. Because dop-3 is expressed in many neurons (Chase et al., 2004) (K.F. and K.D.K., data not shown), we used neuronal promoters that could potentially overlap with the dop-3 gene expression. Although dop-3 functions in motor neurons to regulate locomotion (Chase et al., 2004), expression in motor neurons with acr-2 or unc- 47 promoters did not rescue the phenotype (Fig. 7A). Effective rescue of the phenotype was observed only with the expression by the panneuronal H20 promoter and by the cell-specific lin-11 and $t b h-1$ promoters (Fig. 7A). The expression of lin-11 and tbh-1 promoters overlap only in a pair of RIC interneurons (Hobert et al., 1998; Alkema et al., 2005), and the other cell-specific promoters are not expressed in the RIC neurons (Fig. 7A). Consistently, dop-3 was expressed in the RIC neurons (Suo et al., 2009) (K.F. and K.D.K., data not shown). Therefore, these results suggest that the RIC neurons are at least one of the major sites-of-action for $d o p$ - 3 to regulate the enhancement of 2-nonanone avoidance. 
The RIC neurons are known to be octopaminergic (Alkema et al., 2005), and it has been suggested that dop-3 activity in the RIC neurons suppresses octopamine release from the cells (Suo et al., 2009). To test this possibility in the regulation of 2-nonanone avoidance, we analyzed the phenotype of the $t b h-1$ single mutants and the dop$3 t b h-1$ double mutants; tbh-1 encodes a tyramine $\beta$-hydroxylase homolog required for octopamine biosynthesis (Alkema et al., 2005). We found that the defect in the enhancement of 2-nonanone avoidance in the dop-3 mutants was suppressed in the dop$3 t b h-1$ double mutants (Fig. $7 B$ ). This supports the idea that octopamine from the RIC neurons functions antagonistically downstream of dopamine signaling. Although the dop-3tbh-1 double mutant showed significant enhancement after preexposure, its magnitude was statistically different from that of the wild-type animals (Fig. $7 B$, lanes 3 vs $12, p<0.001$ ), suggesting that the antagonistic octopamine signaling may not be the only output from dopamine signaling to regulate the enhancement (see Discussion).

It has been shown that $d o p-3$ functions also in the SIA interneurons and that dop-3 activity in the RIC and SIA neurons suppresses the starvation-induced cAMP response element-binding protein (CREB) activation in the SIA, although the physiological role of the CREB activation is not yet known (Suo et al., 2009). For the enhancement of 2-nonanone avoidance, however, the expression of $d o p-3$ in the SIA neurons did not rescue the phenotype (Fig. $7 A$, lanes 13-15), and mutation in the CREB homolog crh-1 did not suppress the $d o p-3$ phenotype (Fig. $7 C$ ), suggesting that $d o p-3$ signaling in the SIA neurons is not involved in the enhancement. Additional experiments may be required to further confirm these results.

\section{Discussion}

Animals modulate their behavior on the basis of prior experience, which should play critical roles in survival and/or reproduction. We have demonstrated that $C$. elegans exhibit the enhancement of odor avoidance after preexposure to a repulsive odor, which may be beneficial for the animals to protect themselves from further disturbance (see below). We also showed that the enhancement of 2-nonanone avoidance is caused after the preexposure in the presence and absence of food and that it is regulated by dopamine signaling via the $\mathrm{D}_{2}$-like dopamine receptor DOP-3 in the RIC interneurons. These data suggest a new role of dopamine signaling to regulate experience-dependent neural function in $C$. elegans.

\section{Enhancement of odor avoidance caused by nonassociative conditioning}

C. elegans has been shown to exhibit learning in associative as well as nonassociative forms (Torayama et al., 2007; for review, see Giles et al., 2006). Our data suggest that the enhancement of 2-nonanone avoidance is classified as nonassociative learning. Nonassociative learning results when an animal is exposed once or repeatedly to a single type of stimulus (Kandel et al., 2000), and the enhancement of 2-nonanone avoidance is caused by preexposure to 2-nonanone. The enhancement does not require the presence or absence of food, which generally functions as a strong positive or negative unconditioned stimulus, respectively, or other unconditioned stimulus as in the case of odor associative learning with aversive acetic acid solution (Morrison et al., 1999). Among nonassociative forms of learning in C. elegans, reduction of sensory responses such as adaptation or habituation have been reported in many sensory responses. In contrast, the enhancement of sensory response in nonassociative form has been reported only in tap response (Rankin et al., 1990), which has not been studied further. Therefore, the enhancement of 2-nonanone avoidance may provide a paradigm to study a new type of nonassociative behavioral plasticity in C. elegans.

Enhancement of sensory responses in nonassociative form to a noxious stimulus has been reported in other animals as sensitization. Well-known examples are the sensitization of mammalian peripheral pain sensation and of the defensive response in the leech and Aplysia (Hawkins et al., 1993; Sahley, 1995; Millan, 1999). In addition, sensitization to the tap stimulus in C. elegans is also induced by a stronger stimulus of a train of taps (Rankin et al., 1990). Interestingly, the enhancement of 2-nonanone avoidance in C. elegans is also caused by a potentially noxious stimulus because the higher concentration of 2-nonanone used in the preexposure (90 nl/plate) is close to the toxic threshold: preexposure with much higher concentration (300 nl per plate) made the animals sick and almost immobile (K.D.K. and I.K., data not shown). Sensitization is generally regarded as an enhanced re- 

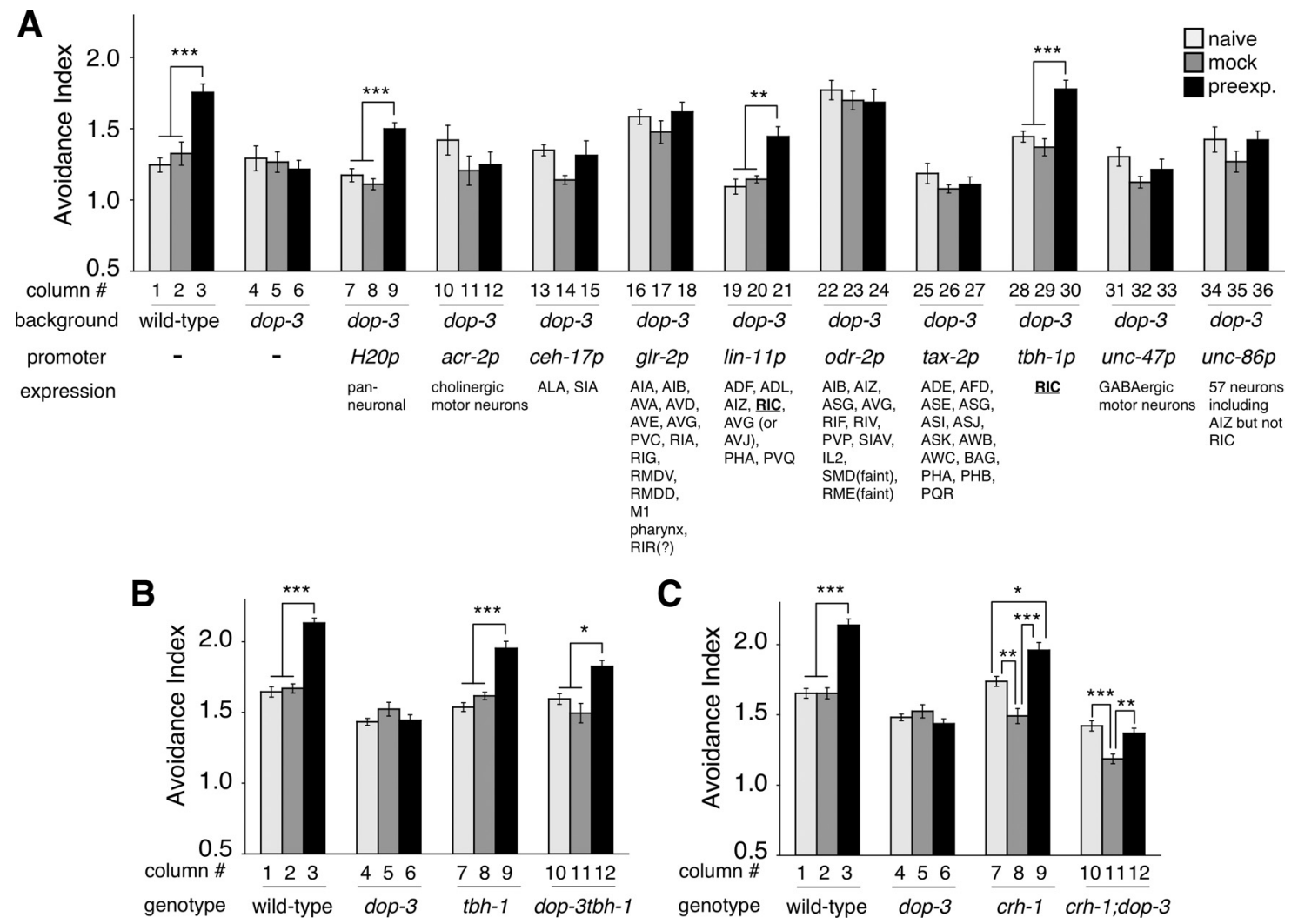

Figure 7. dop-3 dopamine receptor homolog functions in the RIC interneurons to regulate the enhancement. $\boldsymbol{A}$, Cell-specific rescue experiments of the enhancement of 2 -nonanone avoidance in the dop-3 mutant. A plasmid containing a cell-specific promoter fused to the dop-3 CDNA ( $5 \mathrm{or} 50 \mathrm{ng} / \mu \mathrm{l}$ ) was introduced into the dop-3(tm 1356 ) mutant background. The transgenic animals were preexposed to $90 \mathrm{nl}$ of 2-nonanone in the absence of food, and then assayed with $600 \mathrm{nl}$ of 2-nonanone. Avoidance behavior of the wild-type and dop-3 mutant animals containing transgene with the marker and the carrier DNA were also analyzed in parallel as controls. H2Op and tbh-1p are the results of transgenic lines with $5 \mathrm{ng} / \mu$ lof the fusion plasmid and the others are the results with $50 \mathrm{ng} / \mu \mathrm{l}$. At least two independent transgenic lines were analyzed for each concentration. Some promoters led to higher or lower basal locomotion activity in the transgenic animals than in the control animals ( $g / r-2 p$ and odr-2p for example), probably due to inappropriate expression of the dop-3 CDNA. The reported expression pattern of each promoter (see Materials and Methods) is shown at the bottom. Each bar represents the mean \pm SEM of 9 (rescued animals) or 6 (non-rescued animals) independent experiments. $\boldsymbol{B}, \boldsymbol{C}$, The behavioral defect in the dop-3 mutants were suppressed by the tbh-1 (B) mutation but not by $\mathrm{crh}-1(\boldsymbol{C})$. The animals were preexposed to $90 \mathrm{nl}$ of 2-nonanone in the absence of food and assayed with $600 \mathrm{nl}$ of 2 -nonanone.

sponse to a wide variety of stimuli after the presentation of an intense or noxious stimulus (Kandel et al., 2000). Although preexposure to 2-nonanone or 1-octanol causes the enhancement of avoidance behavior to either of the odors, the enhancement is not caused in higher osmolarity avoidance. Therefore at this point we do not classify the enhancement of 2-nonanone avoidance as a type of sensitization. Still, the enhancement of 2-nonanone avoidance is likely a new example of nonassociative enhancement of sensory response to a noxious stimulus.

Despite its possible similarity to the noxious stimulusinduced behavioral sensitizations mentioned above, our data suggest that the enhancement of 2-nonanone avoidance is regulated by a different mechanism from the other sensitizations. Pain sensitization is caused by pain-inducing substances released from the neighboring environment, but not by pain itself (Basbaum and Jessell, 2000), while the enhancement of 2-nonanone avoidance is caused by the odor stimulus itself. The sensitization in the defensive responses in the leech and Aplysia are mainly regulated by serotonin (Hawkins et al., 1993; Sahley, 1995), and the enhancement of 2-nonanone avoidance requires dopamine but not serotonin (Fig. 5A). For tap sensitization in C. elegans, a twofold increase in the response is observed as early as a few minutes after the conditioning stimulus (Rankin et al., 1990) while the effect of preexposure takes much longer (30-60 min; Fig. $3 A$ ) to become detectable in 2-nonanone avoidance. These results may suggest an evolutionary convergence of noxious stimulus-induced sen- sory enhancement (Woolf and Walters, 1991) from nematodes to mammals and its significance in animals' neural function.

\section{The enhancement of 2-nonanone avoidance is regulated by dopamine signaling}

To understand the molecular basis for the enhancement of 2-nonanone avoidance, we took a genetic approach, which revealed that dopamine signaling is required for the enhancement. This result is consistent with the neuromodulatory role of dopamine signaling in C. elegans. Dopamine has been known to function as a "presence of food" signal for short periods to regulate various behaviors in C. elegans (Schafer and Kenyon, 1995; Weinshenker et al., 1995; Sawin et al., 2000; Hills et al., 2004; Sanyal et al., 2004; Kindt et al., 2007). In addition, dopamine signaling has recently been found to be involved in associative learning of chemical cue and starvation (Hukema et al., 2008; Voglis and Tavernarakis, 2008) as well as in increased preference to ethanol after conditioning with a high concentration of ethanol in the presence of food (Lee et al., 2009). Although the enhancement of 2-nonanone avoidance requires dopamine signaling, it is likely regulated by a different mechanism from that shown in previous studies because the enhancement does not require the presence or absence of food during conditioning. In addition, since the above-mentioned dopamine signaling is not involved in the enhancement of sensory responses in nonassociative form, 
the enhancement of 2-nonanone avoidance is likely regulated by a novel dopamine signaling mechanism in C. elegans.

Despite their significance in dopamine signaling, the dopamine receptor sites-of-action in regulating $C$. elegans behavior have been identified in only a few cases: $\mathrm{D}_{1}$-like DOP-1 functions in mechanosensory neurons to modulate habituation (Sanyal et al., 2004) and in ventral cord motor neurons to regulate the animal's basal locomotion (Chase et al., 2004). $\mathrm{D}_{2}$-like DOP-3 also functions in the ventral cord motor neurons for locomotion (Chase et al., 2004) and in the ASH polymodal nociceptive sensory neurons to negatively regulate their sensitivity (Ezak and Ferkey, 2010). We found that the expression of $d o p-3$ in the RIC interneurons alone can rescue the enhancement of the 2-nonanone avoidance phenotype; this is the first evidence for the involvement of a C. elegans dopamine receptor in a single pair of interneurons to regulate the animal's behavioral plasticity. This result may be analogous to the neuromodulatory roles of dopamine receptors in the CNS of higher animals (see below).

How does the dopamine receptor DOP-3 regulate neuronal activity? In $C$. elegans, genetic evidence suggests that $\mathrm{D}_{2}$-like DOP-3 activates G $\alpha$ o-like GOA-1, which may lead to suppression of acetylcholine release from the motor neurons (Chase et al., 2004) and of octopamine from the RIC interneurons (Suo et al., 2009). For the enhancement of 2-nonanone avoidance, DOP-3 may also suppress octopamine release from the RIC neurons (Fig. $7 B$ ). In addition, our data did not exclude the possibility that the DOP-3 receptor in the RIC neurons may regulate neuronal activity other than the suppression of octopamine. For example, the RIC neurons have major synaptic output to the AVA neurons (White et al., 1986), which are part of the locomotor control circuit that regulates forward and backward movement (Zheng et al., 1999). Therefore changes in the RIC neuronal activity by the DOP- 3 receptor could modulate the AVA activation pattern, which may cause changes in the run duration of the animals (Fig. 4).

Dopamine, as well as octopamine or its vertebrate equivalent noradrenalin, play neuromodulatory roles in vertebrates and invertebrates. In the mammalian CNS, dopamine regulates various functions including locomotor activity, cognition, emotion and addiction (Missale et al., 1998). In Drosophila, dopaminergic neurons represent the reinforcing properties of an aversive unconditioned stimulus (i.e., electrical shock) in olfactory and visual associative learning (Unoki et al., 2006; Keene and Waddell, 2007), which is analogous to dopamine regulation of fear conditioning in mammals (Pezze and Feldon, 2004). Although the enhancement of 2-nonanone avoidance in C. elegans does not require an external unconditioned stimulus, it could be a primitive form of dopamine-regulated behavioral plasticity caused by an unpleasant experience similar to the examples in flies and rodents. Thus, taking advantage of available tools in C. elegans such as cell-specific RNAi (Esposito et al., 2007) and calcium imaging of the defined small neuronal network (for example, see Kuhara et al., 2008; Chalasani et al., 2010), further analysis of the enhancement of 2-nonanone avoidance may reveal the fundamental mechanism of dopamine signaling in regulating experience-dependent behavioral plasticity in animals.

\section{References}

Alkema MJ, Hunter-Ensor M, Ringstad N, Horvitz HR (2005) Tyramine functions independently of octopamine in the Caenorhabditis elegans nervous system. Neuron 46:247-260.

Avery L, Horvitz HR (1990) Effects of starvation and neuroactive drugs on feeding in Caenorhabditis elegans. J Exp Zool 253:263-270.

Bargmann CI (2006) Chemosensation in C. elegans. In: WormBook (The C. elegans Research Community, ed), doi/10.1895/wormbook.1.123.1, http://www.wormbook.org.

Bargmann CI, Hartwieg E, Horvitz HR (1993) Odorant-selective genes and neurons mediate olfaction in C. elegans. Cell 74:515-527.

Basbaum AI, Jessell TM (2000) The perception of pain. In: Principles of neural science, Ed 4 (Kandel ER, Schwartz JH, Jessell TM, eds), pp 472491. New York: McGraw-Hill.

Brenner S (1974) The genetics of Caenorhabditis elegans. Genetics 77:71-94. Brockie PJ, Madsen DM, Zheng Y, Mellem J, Maricq AV (2001) Differential expression of glutamate receptor subunits in the nervous system of Caenorhabditis elegans and their regulation by the homeodomain protein UNC-42. J Neurosci 21:1510-1522.

Chalasani SH, Kato S, Albrecht DR, Nakagawa T, Abbott LF, Bargmann CI (2010) Neuropeptide feedback modifies odor-evoked dynamics in Caenorhabditis elegans olfactory neurons. Nat Neurosci 13:615-621.

Chao MY, Komatsu H, Fukuto HS, Dionne HM, Hart AC (2004) Feeding status and serotonin rapidly and reversibly modulate a Caenorhabditis elegans chemosensory circuit. Proc Natl Acad Sci U S A 101:15512-15517.

Chase DL, Koelle MR (2007) Biogenic amine neurotransmitters in C. elegans. In: WormBook (The C. elegans Research Community, ed), doi/ 10.1895/wormbook.1.132.1, http://www.wormbook.org.

Chase DL, Pepper JS, Koelle MR (2004) Mechanism of extrasynaptic dopamine signaling in Caenorhabditis elegans. Nat Neurosci 7:1096-1103.

Chou JH, Bargmann CI, Sengupta P (2001) The Caenorhabditis elegans odr-2 gene encodes a novel Ly-6-related protein required for olfaction. Genetics 157:211-224.

Coburn CM, Bargmann CI (1996) A putative cyclic nucleotide-gated channel is required for sensory development and function in C. elegans. Neuron 17:695-706.

Colbert HA, Bargmann CI (1995) Odorant-specific adaptation pathways generate olfactory plasticity in C. elegans. Neuron 14:803-812.

Colosimo ME, Brown A, Mukhopadhyay S, Gabel C, Lanjuin AE, Samuel AD, Sengupta P (2004) Identification of thermosensory and olfactory neuronspecific genes via expression profiling of single neuron types. Curr Biol 14:2245-2251.

Dalton P, Doolittle N, Breslin PA (2002) Gender-specific induction of enhanced sensitivity to odors. Nat Neurosci 5:199-200.

DeZazzo J, Tully T (1995) Dissection of memory formation: from behavioral pharmacology to molecular genetics. Trends Neurosci 18:212-218.

Driscoll M, Chalfie M (1991) The mec-4 gene is a member of a family of Caenorhabditis elegans genes that can mutate to induce neuronal degeneration. Nature 349:588-593.

Duerr JS, Frisby DL, Gaskin J, Duke A, Asermely K, Huddleston D, Eiden LE, Rand JB (1999) The cat-1 gene of Caenorhabditis elegans encodes a vesicular monoamine transporter required for specific monoaminedependent behaviors. J Neurosci 19:72-84.

Eastman C, Horvitz HR, Jin Y (1999) Coordinated transcriptional regulation of the unc-25 glutamic acid decarboxylase and the $u n c-47$ GABA vesicular transporter by the Caenorhabditis elegans UNC-30 homeodomain protein. J Neurosci 19:6225-6234.

Esposito G, Di Schiavi E, Bergamasco C, Bazzicalupo P (2007) Efficient and cell specific knock-down of gene function in targeted C. elegans neurons. Gene 395:170-176.

Ezak MJ, Ferkey DM (2010) The C. elegans $\mathrm{D}_{2}$-like dopamine receptor DOP-3 decreases behavioral sensitivity to the olfactory stimulus 1-octanol. PloS One 5:e9487.

Feng Z, Cronin CJ, Wittig JH Jr, Sternberg PW, Schafer WR (2004) An imaging system for standardized quantitative analysis of C. elegans behavior. BMC Bioinformatics 5:115.

Finney M, Ruvkun G (1990) The unc-86 gene product couples cell lineage and cell identity in C. elegans. Cell 63:895-905.

Giles AC, Rose JK, Rankin CH (2006) Investigations of learning and memory in Caenorhabditis elegans. Int Rev Neurobiol 69:37-71.

Gray JM, Hill JJ, Bargmann CI (2005) A circuit for navigation in Caenorhabditis elegans. Proc Natl Acad Sci U S A 102:3184-3191.

Hallam S, Singer E, Waring D, Jin Y (2000) The C. elegans NeuroD homolog cnd-1 functions in multiple aspects of motor neuron fate specification. Development 127:4239-4252.

Hawkins RD, Kandel ER, Siegelbaum SA (1993) Learning to modulate transmitter release: themes and variations in synaptic plasticity. Annu Rev Neurosci 16:625-665.

Hilliard MA, Apicella AJ, Kerr R, Suzuki H, Bazzicalupo P, Schafer WR 
(2005) In vivo imaging of C. elegans ASH neurons: cellular response and adaptation to chemical repellents. EMBO J 24:63-72.

Hills T, Brockie PJ, Maricq AV (2004) Dopamine and glutamate control area-restricted search behavior in Caenorhabditis elegans. J Neurosci 24:1217-1225.

Hobert O, D’Alberti T, Liu Y, Ruvkun G (1998) Control of neural development and function in a thermoregulatory network by the LIM homeobox gene lin-11. J Neurosci 18:2084-2096.

Horvitz HR, Chalfie M, Trent C, Sulston JE, Evans PD (1982) Serotonin and octopamine in the nematode Caenorhabditis elegans. Science 216:1012-1014.

Hukema RK, Rademakers S, Jansen G (2008) Gustatory plasticity in C. elegans involves integration of negative cues and $\mathrm{NaCl}$ taste mediated by serotonin, dopamine, and glutamate. Learn Mem 15:829-836.

Kandel ER, Kupfermann I, Iversen S (2000) Learning and memory. In: Principles of neural science, Ed 4 (Kandel ER, Schwartz JH, Jessell TM, eds), pp 1227-1246. New York: McGraw-Hill.

Keene AC, Waddell S (2007) Drosophila olfactory memory: single genes to complex neural circuits. Nat Rev Neurosci 8:341-354.

Kindt KS, Quast KB, Giles AC, De S, Hendrey D, Nicastro I, Rankin CH, Schafer WR (2007) Dopamine mediates context-dependent modulation of sensory plasticity in C. elegans. Neuron 55:662-676.

Kuhara A, Okumura M, Kimata T, Tanizawa Y, Takano R, Kimura KD, Inada H, Matsumoto K, Mori I (2008) Temperature sensing by an olfactory neuron in a circuit controlling behavior of C. elegans. Science 320:803-807.

Lee J, Jee C, McIntire SL (2009) Ethanol preference in C. elegans. Genes Brain Behav 8:578-585.

Lints R, Emmons SW (1999) Patterning of dopaminergic neurotransmitter identity among Caenorhabditis elegans ray sensory neurons by a TGFbeta family signaling pathway and a Hox gene. Development 126:5819-5831.

Liu DW, Thomas JH (1994) Regulation of a periodic motor program in C. elegans. J Neurosci 14:1953-1962.

Loer CM, Kenyon CJ (1993) Serotonin-deficient mutants and male mating behavior in the nematode Caenorhabditis elegans. J Neurosci 13:5407-5417.

Mello CC, Kramer JM, Stinchcomb D, Ambros V (1991) Efficient gene transfer in C. elegans: extrachromosomal maintenance and integration of transforming sequences. EMBO J 10:3959-3970.

Millan MJ (1999) The induction of pain: an integrative review. Prog Neurobiol 57:1-164.

Missale C, Nash SR, Robinson SW, Jaber M, Caron MG (1998) Dopamine receptors: from structure to function. Physiol Rev 78:189-225.

Morrison GE, Wen JY, Runciman S, van der Kooy D (1999) Olfactory associative learning in Caenorhabditis elegans is impaired in $l r n-1$ and $l r n-2$ mutants. Behav Neurosci 113:358-367.

Pezze MA, Feldon J (2004) Mesolimbic dopaminergic pathways in fear conditioning. Prog Neurobiol 74:301-320.

Pierce-Shimomura JT, Morse TM, Lockery SR (1999) The fundamental role of pirouettes in Caenorhabditis elegans chemotaxis. J Neurosci 19:9557-9569.

Ranganathan R, Sawin ER, Trent C, Horvitz HR (2001) Mutations in the Caenorhabditis elegans serotonin reuptake transporter MOD-5 reveal serotonin-dependent and -independent activities of fluoxetine. J Neurosci 21:5871-5884.

Rankin CH, Beck CD, Chiba CM (1990) Caenorhabditis elegans: a new model system for the study of learning and memory. Behav Brain Res 37:89-92.

Rose JK, Rankin CH (2001) Analyses of habituation in Caenorhabditis elegans. Learn Mem 8:63-69.

Sahley CL (1995) What we have learned from the study of learning in the leech. J Neurobiol 27:434-445.

Sanyal S, Wintle RF, Kindt KS, Nuttley WM, Arvan R, Fitzmaurice P, Bigras E, Merz DC, Hébert TE, van der Kooy D, Schafer WR, Culotti JG, Van Tol HH (2004) Dopamine modulates the plasticity of mechanosensory responses in Caenorhabditis elegans. EMBO J 23:473-482.

Sawin ER, Ranganathan R, Horvitz HR (2000) C. elegans locomotory rate is modulated by the environment through a dopaminergic pathway and by experience through a serotonergic pathway. Neuron 26:619-631.

Schafer WR, Kenyon CJ (1995) A calcium-channel homologue required for adaptation to dopamine and serotonin in Caenorhabditis elegans. Nature 375:73-78.

Sugiura M, Fuke S, Suo S, Sasagawa N, Van Tol HH, Ishiura S (2005) Characterization of a novel $\mathrm{D}_{2}$-like dopamine receptor with a truncated splice variant and a $\mathrm{D}_{1}$-like dopamine receptor unique to invertebrates from Caenorhabditis elegans. J Neurochem 94:1146-1157.

Sulston J, Hodgkin J (1988) Methods. In: The nematode Caenorhabditis elegans (Wood WB, ed), pp 587-606. Plainview, NY: Cold Spring Harbor Laboratory.

Suo S, Culotti JG, Van Tol HH (2009) Dopamine counteracts octopamine signalling in a neural circuit mediating food response in C. elegans. EMBO J 28:2437-2448.

Suzuki H, Kerr R, Bianchi L, Frøkjaer-Jensen C, Slone D, Xue J, Gerstbrein B, Driscoll M, Schafer WR (2003) In vivo imaging of C. elegans mechanosensory neurons demonstrates a specific role for the MEC-4 channel in the process of gentle touch sensation. Neuron 39:1005-1017.

Sze JY, Victor M, Loer C, Shi Y, Ruvkun G (2000) Food and metabolic signalling defects in a Caenorhabditis elegans serotonin-synthesis mutant. Nature 403:560-564.

Thompson RF, Spencer WA (1966) Habituation: a model phenomenon for the study of neuronal substrates of behavior. Psychol Rev 73:16-43.

Tomioka M, Adachi T, Suzuki H, Kunitomo H, Schafer WR, Iino Y (2006) The insulin/PI 3-kinase pathway regulates salt chemotaxis learning in Caenorhabditis elegans. Neuron 51:613-625.

Torayama I, Ishihara T, Katsura I (2007) Caenorhabditis elegans integrates the signals of butanone and food to enhance chemotaxis to butanone. J Neurosci 27:741-750.

Troemel ER, Kimmel BE, Bargmann CI (1997) Reprogramming chemotaxis responses: sensory neurons define olfactory preferences in C. elegans. Cell 91:161-169.

Unoki S, Matsumoto Y, Mizunami M (2006) Roles of octopaminergic and dopaminergic neurons in mediating reward and punishment signals in insect visual learning. Eur J Neurosci 24:2031-2038.

Voglis G, Tavernarakis N (2008) A synaptic DEG/ENaC ion channel mediates learning in C. elegans by facilitating dopamine signalling. EMBO J 27:3288-3299.

Waddington JL, O’Boyle KM (1989) Drugs acting on brain dopamine receptors: a conceptual re-evaluation five years after the first selective D-1 antagonist. Pharmacol Ther 43:1-52.

Wang HW, Wysocki CJ, Gold GH (1993) Induction of olfactory receptor sensitivity in mice. Science 260:998-1000.

Weinshenker D, Garriga G, Thomas JH (1995) Genetic and pharmacological analysis of neurotransmitters controlling egg laying in C. elegans. J Neurosci 15:6975-6985.

White JG, Southgate E, Thomson JN, Brenner S (1986) The structure of the nervous system of the nematode Caenorhabditis elegans. Philos Trans R Soc Lond B Biol Sci 314:1-340.

Wicks SR, de Vries CJ, van Luenen HG, Plasterk RH (2000) CHE-3, a cytosolic dynein heavy chain, is required for sensory cilia structure and function in Caenorhabditis elegans. Dev Biol 221:295-307.

WoolfCJ, Walters ET (1991) Common patterns of plasticity contributing to nociceptive sensitization in mammals and Aplysia. Trends Neurosci 14:74-78.

Wysocki CJ, Dorries KM, Beauchamp GK (1989) Ability to perceive androstenone can be acquired by ostensibly anosmic people. Proc Natl Acad Sci U S A 86:7976-7978.

Yee KK, Wysocki CJ (2001) Odorant exposure increases olfactory sensitivity: olfactory epithelium is implicated. Physiol Behav 72:705-711.

Zheng Y, Brockie PJ, Mellem JE, Madsen DM, Maricq AV (1999) Neuronal control of locomotion in C. elegans is modified by a dominant mutation in the GLR-1 ionotropic glutamate receptor. Neuron 24:347-361. 